\title{
WiCV 2018: The Fourth Women In Computer Vision Workshop
}

\author{
Ilke Demir ${ }^{1}$, Dena Bazazian ${ }^{2}$, Adriana Romero $^{3}$, Viktoriia Sharmanska ${ }^{4}$, Lyne P. Tchapmi ${ }^{5}$ \\ ${ }^{1}$ Facebook, ${ }^{2}$ Computer Vision Center, ${ }^{3}$ Facebook AI Research, \\ ${ }^{4}$ Imperial College London, ${ }^{5}$ Stanford University
}

\begin{abstract}
We present WiCV 2018 - Women in Computer Vision Workshop to increase the visibility and inclusion of women researchers in computer vision field, organized in conjunction with CVPR 2018. Computer vision and machine learning have made incredible progress over the past years, yet the number of female researchers is still low both in academia and industry. WiCV is organized to raise visibility of female researchers, to increase the collaboration, and to provide mentorship and give opportunities to femaleidentifying junior researchers in the field. In its fourth year, we are proud to present the changes and improvements over the past years, summary of statistics for presenters and attendees, followed by expectations from future generations.
\end{abstract}

\section{Introduction}

Computer vision has made tremendous progress in the recent years over a wide range of areas, including object recognition, object detection, image understanding, video analysis, 3D reconstruction, autonomous driving, and other domains. It has also become one of the largest computer science research communities. However, despite the expansion of our field, the percentage of female researchers both in academia and in industry is still relatively low. As a result, most female computer vision researchers feel isolated and the lack of inclusion creates unbalanced workspaces.

The workshop on Women in Computer Vision is a gathering for both women and men working in computer vision targeting a broad and diverse audience of researchers, including graduate students pursuing masters or doctoral studies, exceptional undergraduates interested in research, and researchers in both industry and academia (faculty, postdocs, etc). This workshop is envisioned as a unique opportunity to raise the profile and visibility of female computer vision researchers at all levels, seeking to serve women from universities, research programs and backgrounds from all around the world. We encourage both male and female researchers to attend the workshop.
The first goal of the workshop is to raise visibility of women in computer vision. We accomplish this goal by inviting high quality research talks from junior and senior female researchers to present their work as keynote speakers and oral presentations. The presentations of junior researchers will be of novel or recently published work. We also strive for diversity in the selected research topics and presenters' backgrounds. The main goal of these sessions is to give female students an opportunity to give a research talk in a professional and supportive setting. The keynote presentations help us suggest role-models in our area whom newcomers can both have a neutral ground to collaborate with and follow their steps in their career path. The workshop also includes a panel, where inclusion and diversity topics can be discussed in an open and friendly environment between female and male colleagues.

Our second goal of giving opportunities to junior female students and researchers is accomplished by enabling them to present their work via a poster session, and providing travel awards to ease and encourage their participation. We invite three page short papers on current or previously published work from female researchers, which is then presented during the poster session. We particularly encourage work in progress and work from junior graduate students (or exceptional undergraduate students who are interested in research) so that the authors have a chance to hear feedback and suggestions on their work.

The last main goal is maintaining and growing WiCV network where female students and professionals share experiences and career advice. We especially organize a mentoring banquet to provide a safe and casual environment for those relationships to be seeded in the workshop, to become ongoing mentorships or collaborations in future. The dinner sets an informal setting in which junior women can meet, exchange ideas, and form beneficial relationships with senior faculty and researchers in the field.

\section{Updates and News}

WiCV was held for the first time at CVPR 2015. It had great participation with similar number of participants for the 2016 edition and an increased participation of $30 \%$ for 
2017. Over the years, the attendance and quality of submissions to WiCV significantly increased, which encouraged us to collect the submissions into workshop proceedings. We believe that this year we took a big step towards increasing visibility of female researchers by having workshop proceedings. In addition to a temporary poster presentation, we enable them to share their research with a permanent publication which can reach to members of the community that were not able to attend the workshop.

Compared to previous years, we increased the duration of the workshop from half a day to a full day gathering, inviting more senior and junior researchers to present their work, while still hosting poster presentations to share with the rest of CVPR. Another advance compared to previous years is that the frequency of WiCV has been doubled: in addition to this fourth WiCV edition at CVPR 2018, the fifth WiCV gathering will also be organized this year collocated with ECCV 2018.

\section{Workshop Statistics}

To begin with, we had an incredible set of organizers from geographically distributed institutions at 4 different timezones, with a maximum of 9 hours difference. We had $40-60 \%$ of industry versus academia organizers, all of whom were born in a different country. Moreover, our research areas of interest were also diverse, including computer vision, computer graphics, machine learning, and robotics, bringing many perspectives to the committee.

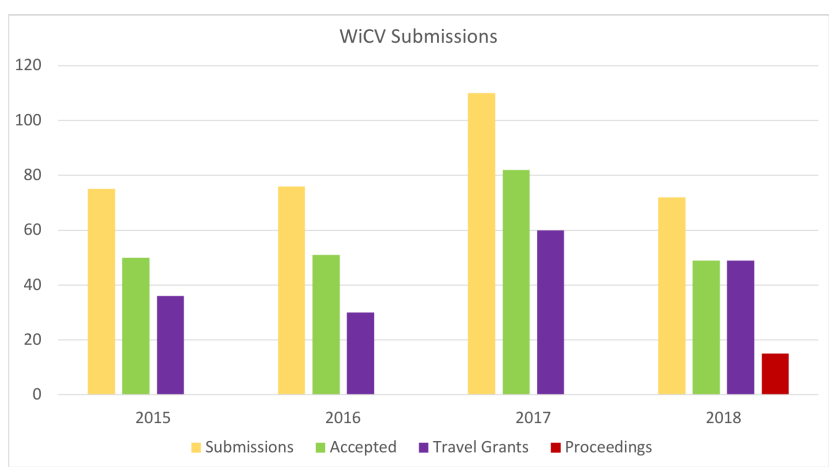

Figure 1: WiCV Submissions. The number of submissions over the past years of WiCV.

This year we had 72 high quality submissions from a diverse range of topics and institutions. The most popular topics were object recognition and detection, applications of computer vision, and machine learning approaches. There were also very interesting research applications ranging from underwater and telescope imaging to detection of animals in the wild. Over all submissions, $8 \%$ were selected to be presented as oral talks, $20.8 \%$ were selected to be included in the proceedings, and $68 \%$ were selected to be pre-

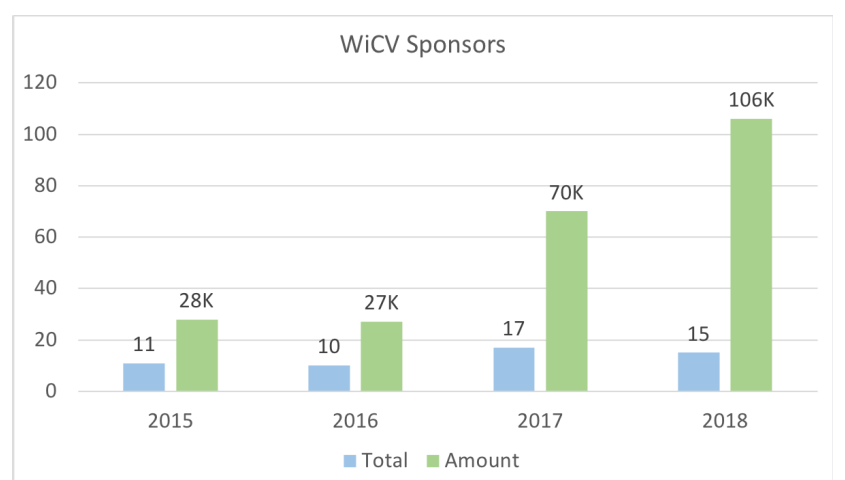

Figure 2: WiCV Sponsors. The progression of number of sponsors and the amount of sponsorships for WiCV.

sented as posters. The comparison with previous years is presented in Figure 1. We had a diverse program committee of 43 reviewers $(61.4 \%$ of our initial reviewer pool) to evaluate and help improve the papers.

Last but not least, we believe that we had a surprising upgrade over past editions, being able provided travel grants for the authors of all accepted submissions who applied for a travel stipend. This is the first time that WiCV is supporting all presenters and we are greatly thankful to our sponsors, who helped us provide opportunities for everyone. We also had a record sponsorship amount of $\$ 106.000$ USD, significantly higher than all previous years' sponsorship amounts as depicted in Figure 2 . The great majority of this sponsorship money will be spent on travel grants; the rest will be used to cover the expenses of the mentorship banquet, workshop bags and promotional materials, in addition to some travel support for keynotes and organizers.

\section{Workshop Program}

We prepared our workshop program to include 4 keynotes, 6 oral presentations, 43 poster presentations, and a panel discussion. This year, we selected our keynote speakers to balance academia versus industry, as well as junior versus senior researchers ratios. Moreover, we put special effort to diversify speakers covering different research domains and backgrounds. We believe that diversifying the set of speakers is of crucial importance to provide junior researchers with potential role models with whom they can identify and who can help them envision their own career paths. This also helps us put all perspectives together in our panel discussion. The details of the workshop are given in the following schedule:

- 08:50: Welcome

- 09:00: Invited Talk 1

- 09:30: Invited Talk 2 
- 10:00: Poster Session and Morning Break

- 11:30: On the iterative refinement of densely connected representation levels for semantic segmentation [3], Arantxa Casanova (MILA)

- 11:50: ARC: Adversarial Robust Cuts for SemiSupervised and Multi-Label Classification [2], Sima Behpour (University of Illinois)

- 12:10: Lunch

- 13:30: Invited Talk 3

- 14:00: Don't Just Assume; Look and Answer: Overcoming Priors for Visual Question Answering [1], Aishwarya Agrawal (Georgia Institute of Technology)

- 14:20: Learnable PINs: Cross-Modal Embeddings for Person Identity [5], Arsha Nagrani (Oxford University)

- 14:50: Invited Talk 4

- 15:20: Gradient-free policy architecture search and adaptation [4], Sayna Ebrahimi (UC Berkeley)

- 15:40: Joint Event Detection and Description in Continuous Video Streams [6], Huijuan Xu (Boston University)

- 16:00: Afternoon Break

- 16:30: Panel

- 17:10: Closing Remarks

\section{Conclusions}

We believe that WiCV 2018 will be a great accomplishment for presenters, participants and organizers towards a more connected community to overcome the ongoing imbalance in the workforce and its side effects. With first-time proceedings, record amount of sponsorships, and a full day gathering, we foresee that the workshop will proudly make its mark towards its goal of increasing visibility, providing support, and building community.

This year we also initiated the plans to convert the WiCV structure to a non-profit organization to be an autonomous entity for all related events and gatherings. We would like to thank all senior researchers that supported this initiative and we are expecting the future generations to carry this torch for a more established community.

\section{Acknowledgments}

We would like to thank our sponsors for seeing the hope in us and being generous. Our banquet sponsor Facebook allows us to plan a wonderful mentorship dinner. We are also grateful to our other Platinum sponsors: Apple, Intel, Google, and NVIDIA. We would also like to acknowledge our Gold sponsors DeepMind, Toyota, IBM, and Uber for their willingness to support. We would also like to thank our Silver Sponsor Lyft, as well as our Bronze sponsors Disney Research, Adobe Research, Snap Inc., Twitter, and Aquifi.

We would like to thank and acknowledge Negar Rostamzadeh for the incredible help and initiative at the beginning of our planning. We would also like to mention that without the information flow and support from the previous $\mathrm{WiCV}$ organizers, this WiCV would not have been possible. We would also like to thank the pioneers of WiCV for mentoring us and answering every possible question we encountered. We would like to also acknowledge CVPR 2018 Workshop Chairs, Mathieu Salzmann and Srikumar Ramalingam for planning and answering all concerns timely, as well as Eric Mortensen for all IEEE publishing questions. Finally, we would like to acknowledge the time and efforts of our program committee, authors, submitters, and our prospective participants for being a part of WiCV.

\section{Contact}

Website: http: / /wicvworkshop.github.io

E-mail: wicv18-organizers@googlegroups.com

Facebook: https://www.facebook.com/ WomenInComputerVision/

Twitter: https://twitter.com/wicvworkshop

\section{References}

[1] A. Agrawal, D. Batra, D. Parikh, and A. Kembhavi. Don't just assume; look and answer: Overcoming priors for visual question answering. 2018.

[2] S. Behpour, W. Xing, and B. D. Ziebart. ARC: Adversarial robust cuts for semi-supervised and multi-label classification. 2018.

[3] A. Casanova, G. Cucurull, M. Drozdzal, A. Romero, and Y. Bengio. On the iterative refinement of densely connected representation levels for semantic segmentation. 2018.

[4] S. Ebrahimi, A. Rohrbach, and T. Darrell. Gradient-free policy architecture search and adaptation. 2018.

[5] A. Nagrani, S. Albanie, and A. Zisserman. Learnable pins: Cross-modal embeddings for person identity. 2018.

[6] H. Xu, B. Li, V. Ramanishka, L. Sigal, and K. Saenko. Joint event detection and description in continuous video streams. 2018. 